\title{
Reliability and validity of a Mediterranean diet and culinary index (MediCul) tool in an older population with mild cognitive impairment
}

\author{
Sue Radd-Vagenas ${ }^{1}$, Maria A. Fiatarone Singh ${ }^{1,2}$, Michael Inskip ${ }^{1}$, Yorgi Mavros ${ }^{1}$, Nicola Gates ${ }^{3}$, \\ Guy C. Wilson ${ }^{1}$, Nidhi Jain ${ }^{1}$, Jacinda Meiklejohn ${ }^{1}$, Henry Brodaty ${ }^{3,4}$, Wei Wen ${ }^{3,5}$, Nalin Singh ${ }^{1}$, \\ Bernhard T. Baune ${ }^{6}$, Chao Suo ${ }^{7}$, Michael K. Baker ${ }^{1,8}$, Nasim Foroughi ${ }^{9}$, Perminder S. Sachdev ${ }^{3,4}$, \\ Michael Valenzuela ${ }^{10}$ and Victoria M. Flood ${ }^{1,11_{*}}$ \\ ${ }^{1}$ Physical Activity, Lifestyle, Ageing and Wellbeing Research Group, Faculty of Health Sciences, The University of Sydney, \\ Lidcombe, NSW 2141, Australia \\ ${ }^{2}$ Hebrew SeniorLife and Jean Mayer USDA Human Nutrition Research Center on Aging at Tufts University, Boston, MA O2111, USA \\ ${ }^{3}$ Centre for Healthy Brain Ageing (CHeBA), School of Psychiatry, University of New South Wales, Randwick, NSW 2031, Australia \\ ${ }^{4}$ Dementia Centre for Research Collaboration, University of New South Wales, Sydney, NSW 2052, Australia \\ ${ }^{5}$ Neuropsychiatric Institute, Prince of Wales Hospital, Randwick, NSW 2031, Australia \\ ${ }^{6}$ Department of Psychiatry, Adelaide Medical School, University of Adelaide, Adelaide, SA 5000, Australia \\ ${ }^{7}$ Brain and Mental Health Hub, Monash Institute of Cognitive and Clinical Neuroscience, School of Psychological Science, \\ Monash University, Clayton, VIC 3800, Australia \\ ${ }^{8}$ School of Exercise Science, Australian Catholic University, Strathfield, NSW 2135, Australia \\ ${ }^{9}$ Clinical and Rehabilitation Research Group, Faculty of Health Sciences, The University of Sydney, Lidcombe, NSW 2141, \\ Australia \\ ${ }^{10}$ Regenerative Neuroscience Group, Brain and Mind Centre and Sydney Medical School, The University of Sydney, \\ Camperdown, NSW 2050, Australia \\ ${ }^{11}$ Western Sydney Local Health District, Westmead Hospital, Westmead, NSW 2145, Australia
}

(Submitted 21 May 2018 - Final revision received 17 July 2018 - Accepted 1 August 2018)

\section{Abstract}

Dementia is a leading cause of morbidity and mortality without pharmacologic prevention or cure. Mounting evidence suggests that adherence to a Mediterranean dietary pattern may slow cognitive decline, and is important to characterise in at-risk cohorts. Thus, we determined the reliability and validity of the Mediterranean Diet and Culinary Index (MediCul), a new tool, among community-dwelling individuals with mild cognitive impairment (MCI). A total of sixty-eight participants (66\% female) aged 75.9 (sD 6.6 ) years, from the Study of Mental and Resistance Training study MCI cohort, completed the fifty-item MediCul at two time points, followed by a 3-d food record (FR). MediCul test-retest reliability was assessed using intra-class correlation coefficients (ICC), Bland-Altman plots and $\kappa$ agreement within seventeen dietary element categories. Validity was assessed against the FR using the Bland-Altman method and nutrient trends across MediCul score tertiles. The mean MediCul score was 54.6/100·0, with few participants reaching thresholds for key Mediterranean foods. MediCul had very good test-retest reliability (ICC $=0.93,95 \% \mathrm{CI} 0.884,0.954, P<0.0001$ ) with fair-to-almost-perfect agreement for classifying elements within the same category. Validity was moderate with no systematic bias between methods of measurement, according to the regression coefficient $(y=-2 \cdot 30+0 \cdot 17 x)(95 \% \mathrm{CI}-0.027,0.358 ; P=0.091)$. MediCul over-estimated the mean FR score by $6 \%$, with limits of agreement being under- and over-estimated by 11 and $23 \%$, respectively. Nutrient trends were significantly associated with increased MediCul scoring, consistent with a Mediterranean pattern. MediCul provides reliable and moderately valid information about Mediterranean diet adherence among older individuals with MCI, with potential application in future studies assessing relationships between diet and cognitive function.

Key words: Validity: Reliability: Mediterranean diet: Index tools

The Mediterranean diet has been associated with many health benefits such as a reduced risk of $\mathrm{CVD}^{(1-4)}$, cancer $^{(5,6)}$, type 2 diabetes $^{(7,8)}$ and neurodegenerative diseases ${ }^{(9,10)}$. The latter includes a slower rate of cognitive decline with age ${ }^{(11)}$, reduced

Abbreviations: ADAS-Cog, Alzheimer's Disease Assessment Scale-cognitive subscale; FR, food record; LOA, limits of agreement; MCI, mild cognitive impairment; MEDAS, Mediterranean Diet Adherence Screener; MediCul, Mediterranean Diet and Culinary Index; SMART, Study of Mental and Resistance Training.

* Corresponding author: Professor V. M. Flood, fax +61293519838, email vicki.flood@sydney.edu.au 
risk of dementia (particularly Alzheimer's disease (AD)) ${ }^{(12)}$ and reduced risk of mild cognitive impairment (MCI) and conversion of MCI to $\mathrm{AD}^{(13)}$. These findings are important as dementia is now a leading cause of morbidity and mortality globally with no pharmacologic options available to prevent, slow or reverse its course $^{(14)}$.

More than thirty Mediterranean diet indexes and their variations ${ }^{(15-19)}$ (including short screeners) ${ }^{(20,21)}$ have been reported in the literature for use in assessing adherence to a Mediterranean dietary pattern. Indexes are popular as they can assess overall dietary patterns, while reducing participant and researcher burden associated with more classical methods of dietary measurement such as long FFQ and weighed food records $(\mathrm{FR})^{(22)}$.

However, limitations exist with the currently available Mediterranean diet indexes. For example, while the original and widely used Mediterranean diet score, and its many iterations ${ }^{(23,24)}$, includes elements determined $a$ priori, the cut-off points used for this tool vary between the populations studied as they are related to mean or median intakes, which may not reflect 'traditional' Mediterranean or optimal intakes. In addition, relatively few Mediterranean diet indexes have been validated directly against an alternate dietary assessment method $^{(20,25,26)}$, especially one not limited by the same recall biases. Further, most Mediterranean diet index scores reported in the literature have been derived indirectly from FFQ (which may or may not be validated), then used to look for associations with health outcomes. Direct validation of dietary tools is now appreciated to be important to reliably interpret results ${ }^{(27)}$.

Importantly, to our knowledge, no existing Mediterranean diet index tools have been validated for use among individuals at various stages of cognitive decline, such as MCI. MCI is considered a pre-dementia stage, defined by subjective concern and mild objective cognitive changes without significant changes in daily functioning related to cognition ${ }^{(28)}$. In terms of prevention, MCI has been identified as a potential window of opportunity for lifestyle or other interventions as approximately $12 \%$ of individuals with MCI convert to AD per year, compared with an annual conversion rate of $1-2 \%$ in the general population $^{(29)}$. It is therefore important to be able to accurately measure adherence to a Mediterranean dietary pattern in older adults who may have already begun to manifest memory difficulties, or are diagnosed with MCI. A Mediterranean diet index tool, which has been validated in such at-risk populations, would be useful for future interventions investigating the potential of nutrition to slow progression of cognitive decline.

A short Spanish Mediterranean Diet Adherence Screener (MEDAS) used in the largest randomised controlled trial of the Mediterranean diet, that is, PREvención con DIeta MEDiterránea (PREDIMED) in cognitively normal but high CVD risk participants, is the only tool, to our knowledge, that has been associated with clinically demonstrated cognitive benefits. In a subcohort of these PREDIMED participants, their MEDAS score out of 14 increased over a 4 -year period by approximately two points from a baseline of $8 \cdot 3-8 \cdot 6^{(19,30)}$. In addition, an increase in MEDAS scoring has been associated with other benefits, such as reduced risk of obesity and breast cancer ${ }^{(6,31)}$. However, it is unclear exactly what the cut-off points in MEDAS mean for cognitive and other health outcomes. Also, the score interpretations for what is considered low, medium and high adherence to the diet in relation to studied outcomes vary for this tool ${ }^{(31,32)}$

In summary, deficiencies in existing Mediterranean diet index tools may reduce their ability to predict health outcomes and guide lifestyle interventions. In addition, no tools have been developed for, and tested in, a cohort with pre-existing cognitive impairment at higher risk of conversion to dementia. Our aim was to test the reliability and validity of a more comprehensive, newly constructed, Mediterranean diet index tool including elements and cut-off points based on the 'traditional' Mediterranean diet, within a cohort of older people with MCI living in a non-Mediterranean country, in order to facilitate clinical research in various at-risk populations.

\section{Methods}

\section{Participants}

We recruited a convenience sample of community-dwelling participants from Sydney, Australia, who fulfilled MCI criteria $^{(29,33)}$ from an existing clinical trial cohort ${ }^{(34)}$, the Study of Mental and Resistance Training (SMART). The flow of participants from the original SMART trial into this validity study can be seen in Fig. 1. SMART participants had been diagnosed with MCI but without dementia, and 100 were randomised between 2008 and 2011 to resistance training and/or cognitive training for 6 months, with follow-up at 18 months and then annually, where possible, to confirm their ongoing cognitive and health status $^{(34)}$. All SMART participants, except those who were deceased, dropped out, uncontactable, involved in piloting the Mediterranean Diet and Culinary Index (MediCul) tool or

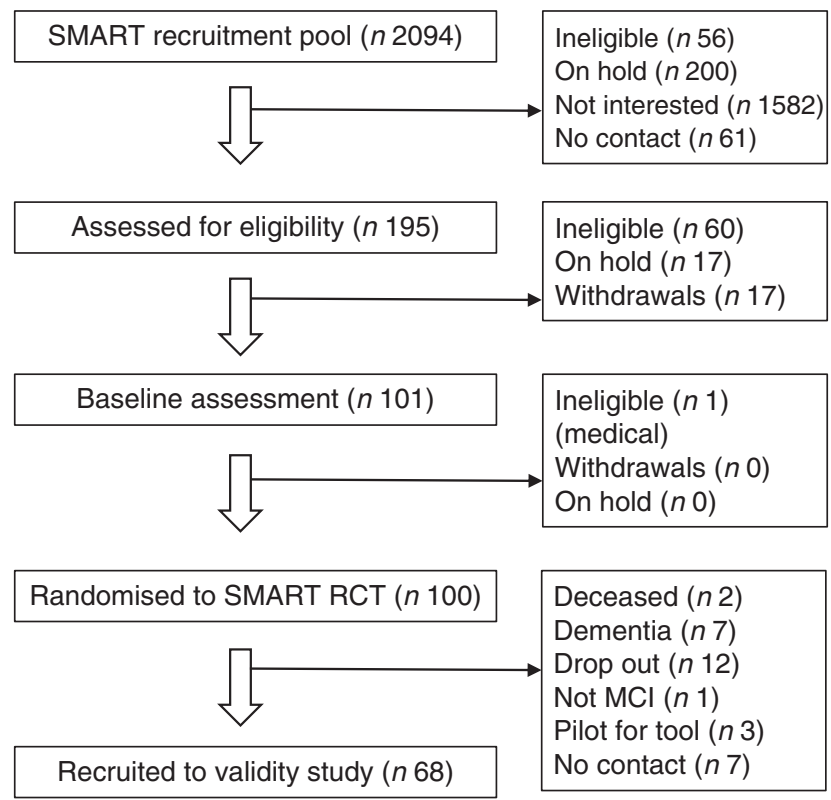

Fig. 1. Participant flow chart. The Study of Mental and Resistance Training (SMART) from which participants were recruited to the Mediterranean Diet and Culinary Index (MediCul) validity study. $n$, number of participants; RCT, randomised controlled trial; $\mathrm{MCl}$, mild cognitive impairment. 
known to have reverted to normal cognition or progressed to dementia were invited to participate in this validity study during one of their annual re-assessment visits, which occurred, on average, 78 months from the time they were originally recruited to SMART with the diagnosis of MCI.

\section{Data administration and collection}

The new Mediterranean diet index tool named MediCul was administered at the University clinic site as a paper survey (survey), twice, 1 week apart (time points $\mathrm{A}$ and $\mathrm{B}$ ), with a dietitian observing and available to clarify questions (S. R.-V.). The dietitian also checked responses to ensure no question was missed. Immediately following survey $\mathrm{B}$, participants were instructed to keep a 3-d FR on any two weekdays and one weekend day within a 7 -d period, representing usual intake. They were asked to specify brands of foods/drinks, preparation methods and recipes, as well as to use the supplied Australian standard household measures (i.e. metric cups, spoons, jug), to estimate quantities. Participants were not required to weigh foods, although some elected to do so. Returned FR were queried with the participant by the dietitian for potentially missed food categories using a checklist.

Anthropometric data were collected at time point A using calibrated digital scales and a wall-mounted stadiometer at the clinic (in light clothing and no shoes) or portable scales and stadiometer (UC-321PBT (A\&D Company Limited) and Seca 213 , respectively) if a home visit was required. BMI was calculated by dividing weight $(\mathrm{kg})$ by height $\left(\mathrm{m}^{2}\right)$.

Additional participant characteristics including education level, marital status, number of chronic diseases, cognitive and physical function scores were sourced from original or followup SMART data, selecting the closest time point available for the entire cohort in our validity study. On average, this was 78 months before the validity study for education and marital status, and 59 months earlier for number of chronic diseases, Alzheimer's Disease Assessment Scale-cognitive subscale (ADAS-Cog), Katz activities of daily living and Bayer informant activities of daily living (Bayer-IADL) scores. ADAS-Cog ${ }^{(35)}$ and Bayer-IADL ${ }^{(36)}$ were used as primary outcomes in SMART for global cognitive function and functional independence, respectively $^{(34)}$.

The study was approved by the Ethics Review Committee (RPAH Zone) of the Sydney Local Health District (Protocol no. X08-0064 \& HREC/08/RPAH/106).

\section{Tool development}

The fifty-item MediCul tool was developed empirically in the form of a short question survey (see online Supplementary Material 1 for elements, cut-off points, scoring and rationale) to (a) reflect a 'traditional' Mediterranean dietary pattern and certain aspects of cuisine not assessed by previous tools ${ }^{(19,37-39)}$, (b) include fourteen questions from the validated MEDAS optimised for the English language ${ }^{(20)}$ and (c) incorporate discretionary foods, commonly consumed in Western populations $^{(40)}$.
After conducting a literature review to identify important Mediterranean dietary elements and existing tools ${ }^{(19)}$, draft questions were developed in consultation with Mediterranean diet and survey tool experts. The tool was pilot-tested with five healthy people from the general public aged 50-80 years and three SMART participants with MCI for readability, ambiguity and completion timing, which is $20 \mathrm{~min}$ on average, before being finalised.

MediCul includes a blend of frequency and serve questions spanning seventeen main elements and assesses their exposure over the past 6 months: olive oil, vegetables, fruit, nuts, whole grains, legumes, fish/shellfish, eggs, dairy products, white meat, $\mathrm{red} /$ processed meats, sweets and sugary drinks, takeaway, water, alcohol, coffee and certain aspects of Mediterranean cuisine. In all, nine of these elements cover desirable features of the 'traditional' Mediterranean diet and four cover undesirable features of a Western diet. MediCul is scored from 0 to 100, with a higher score representing increased adherence to a 'traditional' dietary pattern (online Supplementary Material 2).

\section{Nutritional analysis}

Scoring for both the MediCul and MEDAS tools was operationalised using Excel (MS Office Professional Plus 2013). The FR were coded and entered into FoodWorks 8 Professional Edition: 8.0.3553 (Xyris Software Pty Ltd) selecting AusBrands 2015 and AusFoods 2015 data sources, which map to the AUSNUT 2011-2013 Food Standards Australia New Zealand nutrient database for analysis by S. R.-V. Average intakes for food group outputs were adjusted manually, where required, as FoodWorks draws on the concept of USDA Food Patterns Equivalents Database, which is sometimes contrary to current nutrition guidelines that also consider diet quality (e.g. hot chips are counted in the vegetable group). Missing foods that have become popular in recent times (e.g. paleo bread) were entered into FoodWorks using data from nutrition panels on packaging, and by basing such foods on similar products. Nutrient intakes from supplements were not included, as the aim was to test validity of MediCul based on foods alone ${ }^{(41)}$.

\section{Statistical analysis}

The Statistical Package for Social Sciences for Windows version 24 (SPSS Inc.) was used for all analyses. We aimed to have a minimum of fifty participants as recommended by Peat ${ }^{(42)}$ for adequate assessment of repeatability and agreement.

The distribution of MediCul scores, nutrients and food groups was examined for plausibility with the aid of histograms and by considering minimum and maximum values, to identify potential data entry errors. We did not use cut-offs for potential outliers as we were testing the tool among MCI participants and did not want to exclude for possible cognitive influences in reporting.

A comparison was made of MediCul and the derived MEDAS scores, relevant to cognitive outcomes reported in the literature $^{(30)}$. In addition, we estimated the percentage of MCI participants who reached Mediterranean diet thresholds for selected foods/aspects of 'traditional' cuisine presumed to be 
health promoting, and those rarely used traditionally but consumed at significant levels in Western populations and known to be harmful at high or frequent levels of exposure. This was performed using cut-off points for the highest score for relevant questions, from the MediCul tool (online Supplementary Material 1).

Reliability for MediCul across the two administrations, 1 week apart, was assessed using the intra-class correlation coefficient (ICC) and classified as poor $(<0 \cdot 40)$, fair to good $(\geq 0.4$ and $<0.75)$ and very good $(\geq 0.75)^{(43)}$. A Bland-Altman plot was used to assess the level of agreement between survey A and B time points, as a high correlation does not necessarily mean good agreement ${ }^{(44)}$. $\kappa$ was also used to check percentage agreement within the same category for the seventeen dietary elements. The $\kappa$ values were characterised as showing almost perfect agreement $(0 \cdot 81-1 \cdot 00)$, substantial agreement $(0 \cdot 61-$ $0 \cdot 80)$, moderate agreement $(0 \cdot 41-0 \cdot 60)$, fair agreement $(0 \cdot 21-$ $0 \cdot 40)$, slight agreement $(0 \cdot 00-0 \cdot 20)$ and poor agreement $(<0 \cdot 00)^{(45)}$.

Validity was assessed by comparing MediCul scores derived from survey A $v$. MediCul scores from the FR using the BlandAltman method. The differences between the two methods were plotted against the means of the methods, with limits of agreement (LOA) as 2SD above, and below, the mean difference. Linear regression analysis was used to indicate the direction of bias and whether it was constant across mean scores.

A total of three questions from MediCul relating to growing own vegetables, main meal eaten alone and fasting frequency were unable to be validated as the FR did not include these details. We chose not to score for napping (traditionally conducted immediately after lunch in the Mediterranean); hence, this question was also not validated. Finally, the validation of the MediCul tool was based on scoring out of 97, whereas the reliability analysis was out of 100 .

Indirect validity was investigated by examining whether MediCul scores were associated with expected trends in nutrient intakes extracted from the FR. Nutrient values from the FR were checked for normal distribution using graphical methods and skewness, and log 10 transformed where positively/negatively skewed, then re-checked for normality to inform the statistical tests to be used. Normally distributed or normalised nutrients from the FR were compared across tertiles of MediCul score derived from both survey A and the FR using parametric tests (one-way ANOVA), whereas non-normally distributed nutrients were analysed using non-parametric tests (Kruskal-Wallis). When the ANOVA $F$ ratio was significant, variances were checked for equality, and Bonferroni's test was applied for equal variances or the Games-Howell post hoc test was applied for unequal variances. In addition, first (linear)- and second (quadratic)-order polynomial contrasts were applied to test for nutrient trends across tertiles, as well as the line of best fit.

Means and standard deviations were calculated from FR values for normally distributed nutrients: kilojoules, protein, fat, fat as percentage energy, saturated fatty acids, SFA as percentage energy, SFA as percentage fat, PUFA as percentage fat, MUFA, MUFA as percentage fat, cholesterol, carbohydrate, carbohydrate as percentage energy, sugars, water, dietary fibre, vitamin $\mathrm{C}$, vitamin $\mathrm{A}, \beta$-carotene, $\mathrm{Na}, \mathrm{K}, \mathrm{Mg}, \mathrm{Fe}$ and $\mathrm{Zn}$. Medians and the interquartile range, representing tertiles 1 and 3 of the MediCul score, were calculated for non-normally distributed nutrients: protein as percentage energy, PUFA, $n-3$ long-chain (LC) PUFA, $\alpha$-linolenic acid, EPA, docosapentaenoic acid, DHA, ratio of MUFA to SFA, ratio of total unsaturated fatty acids to SFA, vitamin E, vitamin $B_{12}$, total folate, Se and alcohol.

Linear regression analysis was undertaken to assess precision of the MediCul tool across ADAS-Cog scores, being an index of global cognition ( $n$ 67).

\section{Results}

We recruited sixty-eight participants from the 100 originally randomised to the SMART trial (Fig. 1). All were included in the reliability study and sixty-five participated in the validity study. A total of two participants did not complete the FR and one had an incomplete FR. The majority of the recruited participants were female (65\%), married/de facto $(56 \%)$ and the primary cook at home (71\%). On average, they were aged 75.9 (sD 6.6) years, overweight $\left(\mathrm{BMI}=27 \cdot 3(\mathrm{sD} 5 \cdot 2) \mathrm{kg} / \mathrm{m}^{2}\right)$, well educated (13 (sD 4) years), had $2 \cdot 8$ (sD 1.6) chronic diseases, good physical function and a confirmed MCI diagnosis based on the most recently available ADAS-Cog scores (Table 1).

The mean MediCul score for survey A was 54.6/100.0 (sD $13 \cdot 0$; range: $32 \cdot 5-85 \cdot 5$ ), with $4.4 \%$ of participants scoring $\geq 80 \cdot 0$. The mean derived MEDAS score for survey A was

Table 1. Characteristics of study participants ( $n$ 68) (Mean values and standard deviations; medians, ranges and percentages)

\begin{tabular}{|c|c|c|c|}
\hline & Mean & & SD \\
\hline Age (years) ${ }^{\star}$ & $75 \cdot 9$ & & $6 \cdot 6$ \\
\hline Female (\%) & 64.7 & & \\
\hline BMI $\left(\mathrm{kg} / \mathrm{m}^{2}\right)^{*}$ & $27 \cdot 3$ & & $5 \cdot 2$ \\
\hline Education level (years) $\dagger$ & $13 \cdot 2$ & & $3 \cdot 7$ \\
\hline Married/de facto $(\%) \dagger$ & $55 \cdot 9$ & & \\
\hline Number of chronic diseases $\ddagger$ & $2 \cdot 8$ & & 1.6 \\
\hline Primary cook at home $(\%)^{\star}$ & $70 \cdot 6$ & & \\
\hline ADAS-Cog score (0-70)‡ & $5 \cdot 2$ & & $2 \cdot 5$ \\
\hline ADAS-Cog $\geq 18(\%)$ & 0.0 & & \\
\hline \multicolumn{4}{|l|}{ Katz ADL score $(0-12) \ddagger$} \\
\hline Median & & 0.0 & \\
\hline Range & & $0.0-0.0$ & \\
\hline \multicolumn{4}{|l|}{ Bayer-IADL score $(1-10) \ddagger, \S$} \\
\hline Median & & 0.1 & \\
\hline Range & & $0.0-0.2$ & \\
\hline Bayer-IADL > 3 (\%) & & 0.0 & \\
\hline
\end{tabular}

ADAS-Cog, Alzheimer's Disease Assessment Scale-cognitive subscale, used as the primary test for global cognitive function in Study of Mental and Resistance Training (SMART, higher scores indicate more impairment; cut-off for dementia is $\geq 18$ ); Katz $A D L, K a t z$ index of independence in activities of daily living (higher scores indicate more impairment; cut-off for significant functional impairment is $>0$ ); Bayer-IADL, Bayer informant activities of daily living (higher scores indicate more impairment; cut-off for significant functional impairment is $>3$ ).

* Assessed at time point $A$ of validity study.

$\dagger$ Assessed at baseline of SMART study ${ }^{(34)}$, on average, 78 months before validity study.

$\ddagger$ Assessed at 18 months of SMART study ${ }^{(34)}$, on average, 59 months before validity study with $n 67$ as missing tests for one participant.

$\S$ Participant score substituted for informant score for $n 3$. 


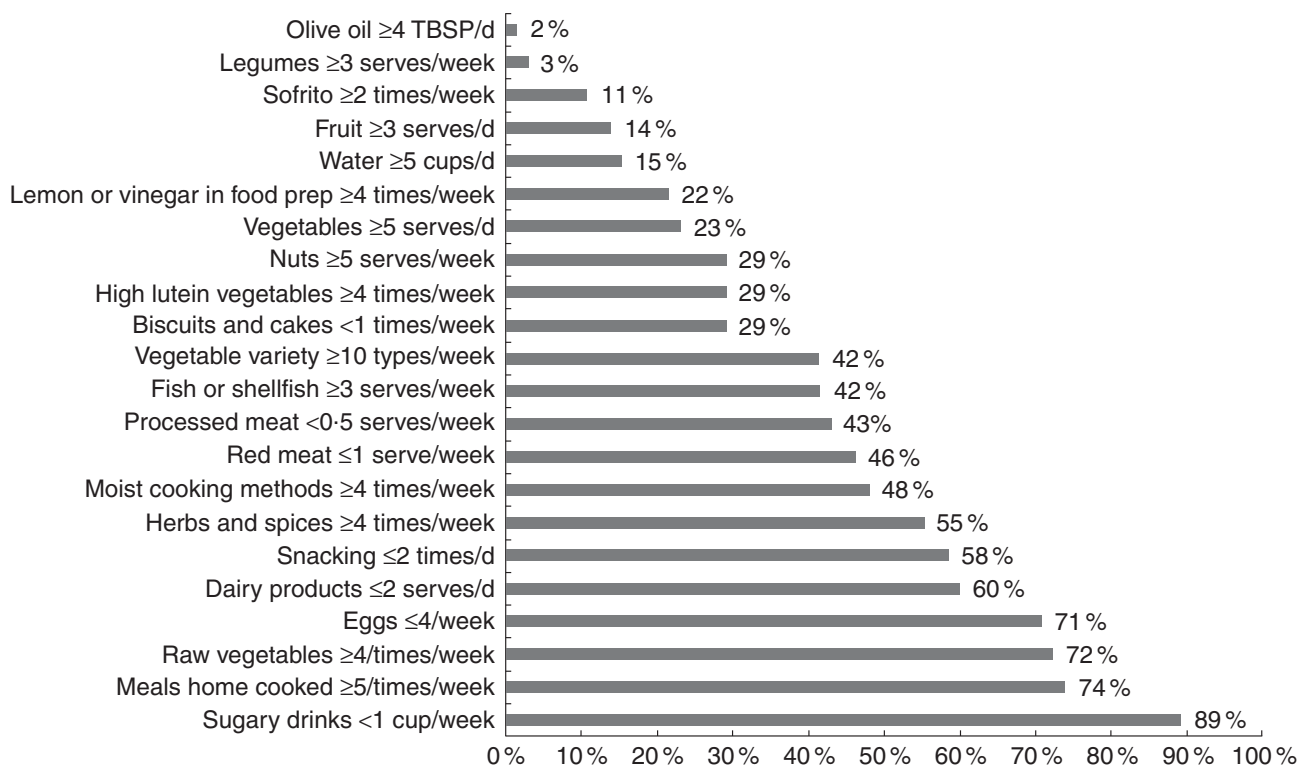

Fig. 2. Percentage of participants who reach Mediterranean diet thresholds according to $3-d$ food records ( $n$ 65). TBSP, tablespoon; prep, preparation.

6.1/14.0 (sD 2.2; range: $1 \cdot 0-11 \cdot 0$ ). All those with MEDAS scores $\geq 10 \cdot 0^{(30)}$ had a MediCul score $\geq 81 \cdot 5$.

On the basis of their FR, few participants reached thresholds for Mediterranean foods considered to be protective for cognitive or vascular function, such as olive oil (2\%), legumes (3\%), fruit (14\%) and water (15\%) (Fig. 2). Fewer than half had minimal exposure to potentially harmful foods, used at low levels in the 'traditional' diet, such as processed meat (43\%) and red meat (46\%). However, three-quarters of the participants did report that they mostly cooked their main meals at home and $89 \%$ kept sugary drinks to a minimum.

\section{Reliability}

The reliability of the MediCul tool, based on single measures and $95 \%$ CI of the ICC, was very good (ICC $=0.93,95 \% \mathrm{CI}$ $0.884,0.954, P<0.0001)$, indicating that the total score was measured similarly at the two time points (A and B). The BlandAltman test for repeated measures showed a mean difference between the two scores of -0.04 , with a lower LOA of -9.7 and an upper LOA of 9.6. In all, sixty-six of the sixty-eight (97\%) participants fell within or on the lower and upper LOA with a fairly even distribution across mean scores. There was also no indication of bias according to the regression coefficient $(y=-0.79+0.01 x)(95 \%$ CI $-0.082,0 \cdot 109 ; P=0.778)$, supporting the null hypothesis that the scores at two time points were equally variable.

Groups that performed well for percentage agreement within the same category at time points A and B were as follows: wholegrains and coffee (almost perfect agreement); fruit, nuts, fish/shellfish, eggs, white meat preference, water and alcohol (substantial agreement); and olive oil, dairy products, $\mathrm{red} /$ processed meats, sweets and sugary drinks (moderate agreement). Groups with fair agreement within the same category were vegetables, legumes, takeaway and cuisine ${ }^{(45)}$
Table 2. Mean difference from paired samples $t$ tests for Mediterranean Diet and Culinary Index (MediCul) scores from surveys A, B, mean AB v. 3-d food record (FR, $n$ 65) (Mean differences and 95\% confidence intervals)

\begin{tabular}{lccc}
\hline & Mean difference & $95 \% \mathrm{Cl}$ & $P$ \\
\hline A v. FR & 5.95 & $3.85,8.05$ & $<0.0001$ \\
B v. FR & 6.29 & $3.95,8.64$ & $<0.0001$ \\
AB v. FR & 6.12 & $3.97,8.28$ & $<0.0001$
\end{tabular}

$A$, first administration of MediCul; B, second administration of MediCul; AB, mean of $A$ and B MediCul administrations.

(online Supplementary Material 3). No groups had poor agreement for the proportion within each category at the two time points.

\section{Validity}

We assessed paired $t$ tests for scores from the survey administered at time points $\mathrm{A}, \mathrm{B}$ and the mean of $\mathrm{AB} v$. the FR $(n 65)$. This analysis indicated a very similar mean difference and CI across the three comparisons, which were all significant $(P<0.0001)$ (Table 2$)$. We therefore used survey A time point for the remaining validity testing, given that this represented first time MediCul use as could be applied in future research.

Bland-Altman analysis showed a positive mean difference of 6.0 between the MediCul score derived from survey A (52.8/ $97 \cdot 0$, SD $12 \cdot 4$, range: $31 \cdot 5-83 \cdot 5)$ and the FR $(46 \cdot 8 / 97 \cdot 0$, SD $10 \cdot 8$, range: $21 \cdot 5-72 \cdot 0)$. Scores for all but one participant fell within or on the $95 \%$ LOA, with a lower LOA of -10.7 and an upper LOA of 22.6. There was also no significant linear trend for the fitted regression line $(y=-2 \cdot 30+0 \cdot 17 x) \quad(95 \%$ CI $-0.027,0 \cdot 358$; $P=0.091$ ), indicating no systematic bias between the two methods of measurement (Fig. 3).

Significant linear trends in relation to tertiles of MediCul score were identified for the following nutrients, consistent 


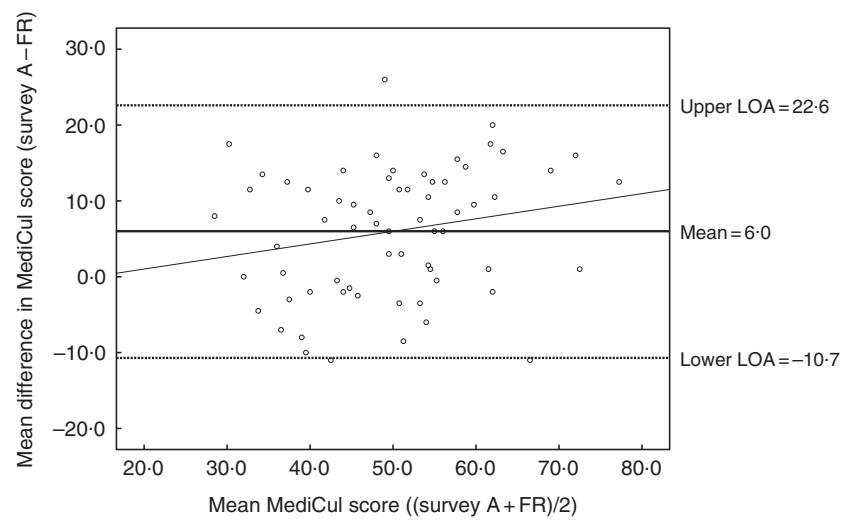

Fig. 3. Bland-Altman plot of the difference between Mediterranean Diet and Culinary Index (MediCul) score measured by survey A (first administration of MediCul) and 3-d food record (FR) and the mean MediCul score of the two methods ( $n$ 65). The solid line in the centre indicates the mean difference between the two methods and the dotted lines above and below indicate the limits within which $95 \%$ of the differences between the methods are expected to fall (2SD above, and below, the mean difference). The fitted regression line is $(y=-2.30+0.17 x) \quad(95 \% \mathrm{Cl}-0.027,0.358 ; \quad P=0.091)$, indicating no systematic bias. LOA, limits of agreement.

with what would be expected for a Mediterranean dietary pattern: total fat (g and \%), including PUFA and MUFA (g), which increased across tertiles of MediCul score from both the survey and FR (Table 3). This also translated into highly significant trends for ratios of MUFA to SFA and total unsaturated fatty acids to SFA $(P<0 \cdot 0001)$. Further, dietary fibre, vitamin $\mathrm{C}$, vitamin $\mathrm{E}$ and $\mathrm{Mg}$ all increased with increasing tertiles of MediCul score from both methods. Conversely, a significant trend for the reduction in carbohydrate as percentage of energy was observed across tertiles of MediCul score from both methods. Protein was either unrelated $(\mathrm{g})$ or significantly decreased (percentage energy) when comparing tertiles from the survey $(P=0 \cdot 041)$, consistent with the fact that a Mediterranean diet is not a high-protein diet. In some instances, there was a trend for nutrients by tertiles of scores from the FR but not the survey (and vice versa) - for example, n-3 LC PUFA. There was no trend observed for total folate. In all cases linear trends were significant, except for $\mathrm{Na}$ when compared with tertiles from the FR, and sugars when compared with tertiles from survey A, where the quadratic trend provided a better fit for the data.

MediCul precision was stable across a range of cognition (from normal ( $\leq 5)$ to MCI (5-12)) using ADAS-Cog as a measure of global cognitive performance.

\section{Discussion}

MediCul is a short survey index tool (takes $20 \mathrm{~min}$ to complete, on average) developed to assess adherence to a 'traditional' Mediterranean dietary pattern and certain aspects of cuisine, within a Western population. On the basis of our analyses, MediCul has very good reliability and moderate validity relative to a FR, among older individuals with MCI. To our knowledge, this is the first Mediterranean diet index tool to be validated in a group at higher risk of dementia. Our results cannot be generalised to younger or cognitively unimpaired individuals without further testing. In addition, our participants were originally volunteers for a randomised clinical trial and well educated, which may have influenced our results. Although we chose not to exclude participants based on extreme energy intakes, these were nevertheless all within plausible limits ${ }^{(46)}$.

The MediCul tool over-estimated the mean total score compared with its reference method by $6 \%$, and this was similar to the findings for MEDAS in Spanish (5\%) and German (9\%) cohorts ${ }^{(20,25)}$. However, it is well known that questionnaires tend to over-estimate intakes compared with $\mathrm{FR}^{(47)}$. Although there was a considerable range for LOA from the Bland-Altman method when comparing scores from the MediCul tool $v$. the FR, and it is unknown whether this may have clinical implications, no systematic bias was found across mean scores. Hence, although the new index tool may be under- and over-estimating the FR-derived MediCul score by 11 and $23 \%$, respectively, the Spanish cohort MEDAS scores were under- and over-estimated by 43 and $53 \%$, respectively, compared with FFQ estimates ${ }^{(20)}$. Further, the under- and over-estimates for the MediCul tool are of a similar range reported for an alternate diet quality index score ${ }^{(48)}$, and well within limits proposed by Ambrosini et al. ${ }^{(49)}$ who classified agreement between an FFQ and FR as being acceptable when LOA were between 50 and $200 \%$.

MediCul captures wide elements of the Mediterranean dietary pattern as a continuous measure. The cut-off points used and nutrient patterns identified suggest that diet quality may be improving with an increased MediCul score. For example, with increasing tertiles of the MediCul score, there is a significant increase in healthy fats (and ratios of MUFA or total unsaturated fats to SFA), as well as dietary fibre, vitamin C and vitamin $\mathrm{E}$, whereas carbohydrate as percentage energy declines correspondingly, and protein remains the same or decreases slightly. These directions are as anticipated for a 'traditional' Mediterranean diet, and macronutrient levels in the third tertile approximate a Mediterranean diet model proposed in Australia ${ }^{(50)}$. For example, the macronutrient proportions in the third tertile of Medicul score from the index tool were as follows: fat, $41 \%$ of energy; protein, $15 \%$ of energy; carbohydrate, $36 \%$ of energy; and MUFA, $47 \%$ of total fat. No trend was observed for total folate, probably a result of fortification in the Australian food supply, making interpretation of folate intakes difficult without additional and specific questions to assess this nutrient.

In our cohort of MCI participants, few reached Mediterranean diet thresholds for adequate intake of certain protective foods, such as olive oil, legumes, fruit and water, and under half met our criterion for high vegetable variety, adequate fish intake and limited red/processed meat intake (Fig. 2). The mean scores from the MediCul tool and the derived MEDAS were also moderately low: 54.6/100.0 (sD 13.0) and 6.1/14.0 (sD $2 \cdot 2$ ), respectively. As a MediCul score of $\geq 81 \cdot 5$ was equivalent to a MEDAS score of $\geq 10 \cdot 0$, a level associated with cognitive benefit in the PREDIMED trial $^{(30)}$, it is of concern that only $3 / 68$ (4.4\%) of older participants with MCI included in our study scored in this range. These findings suggest that individuals with MCI living in Western countries, even those who are well- 
Table 3. Nutrient intakes compared with tertiles of Mediterranean Diet and Culinary Index (MediCul) score $(n$ 65) (Mean values and standard deviations; medians and interquartile ranges (IQR))

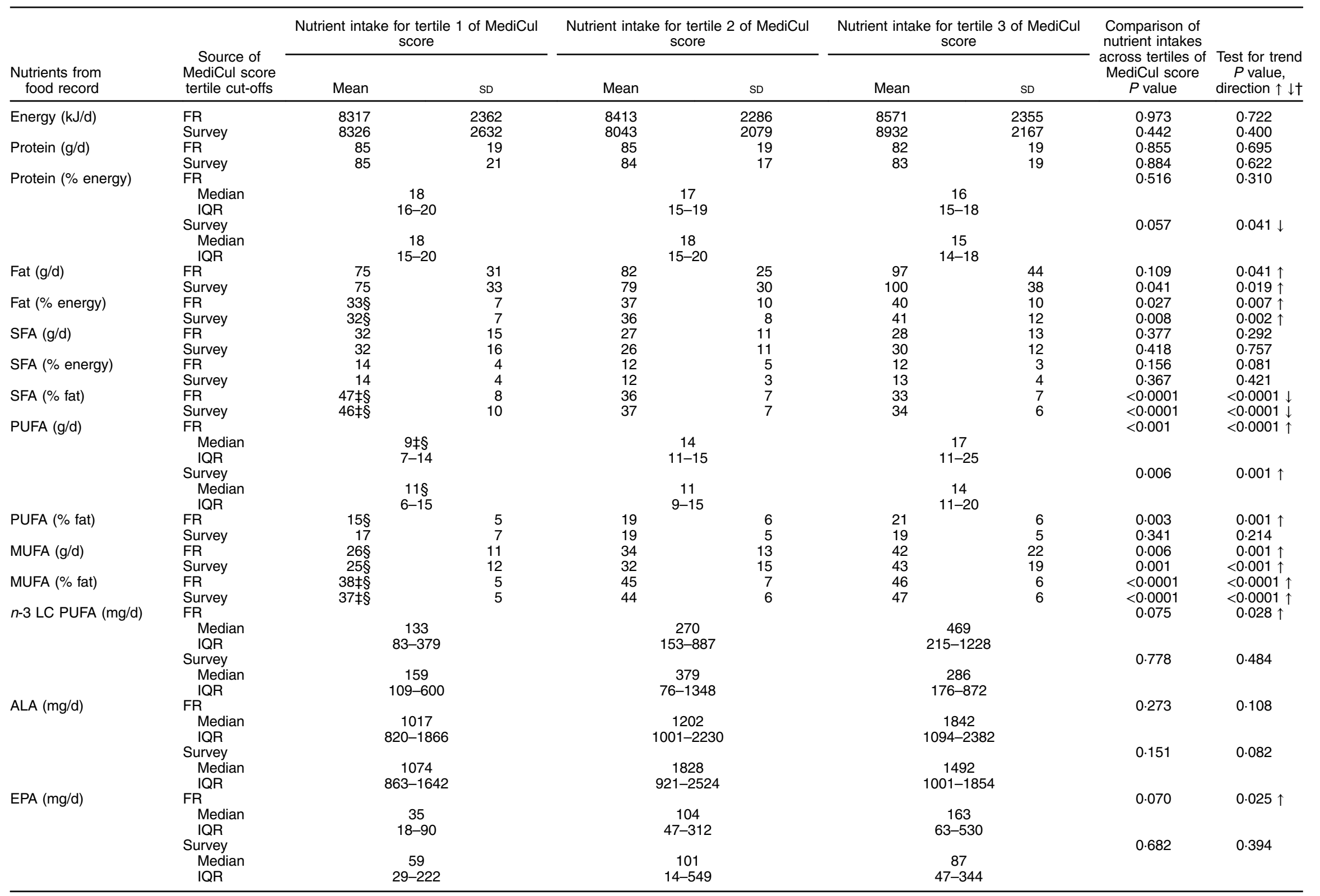


N British Journal of Nutrition

Table 3. Continued

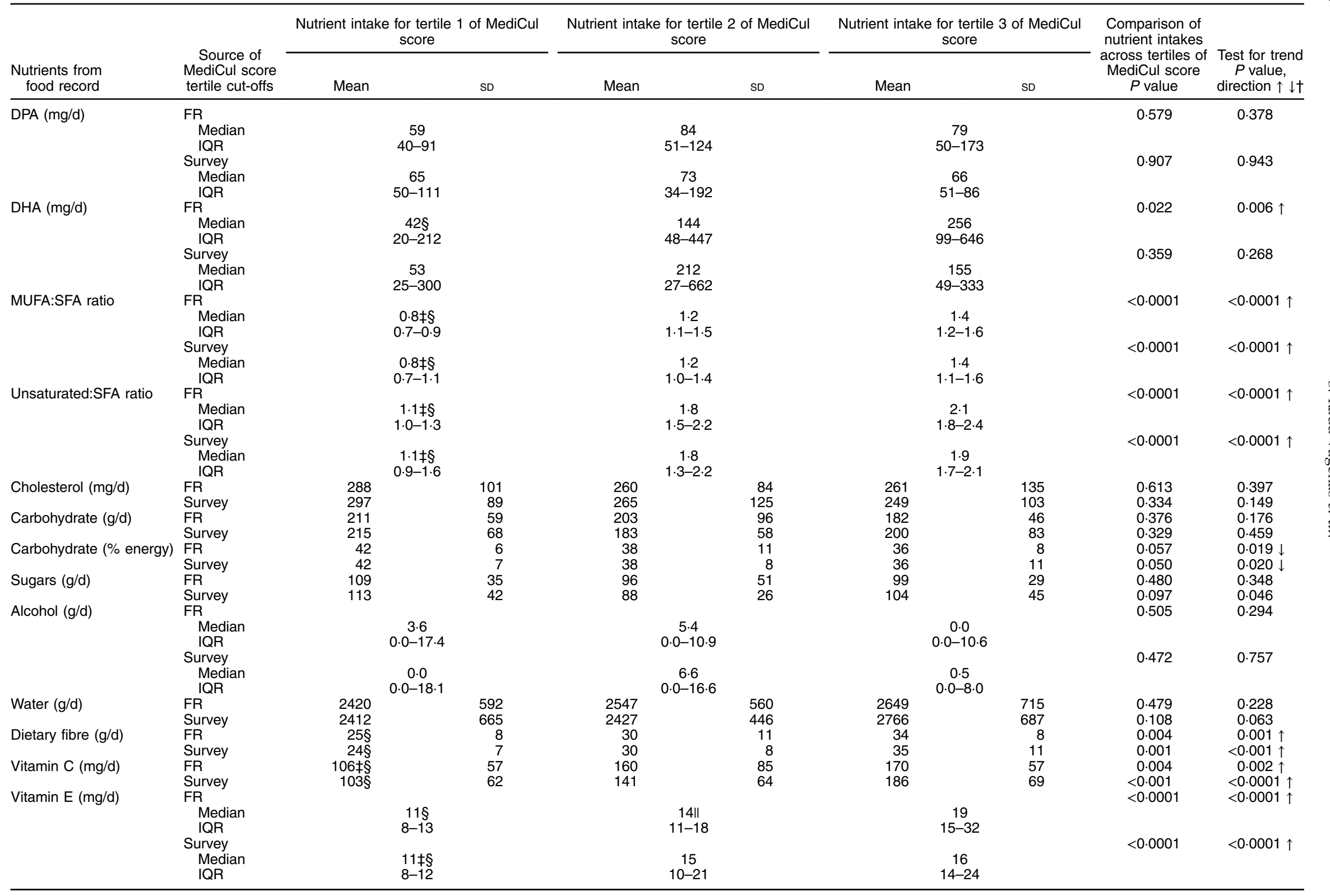




\section{N British Journal of Nutrition}

Table 3. Continued

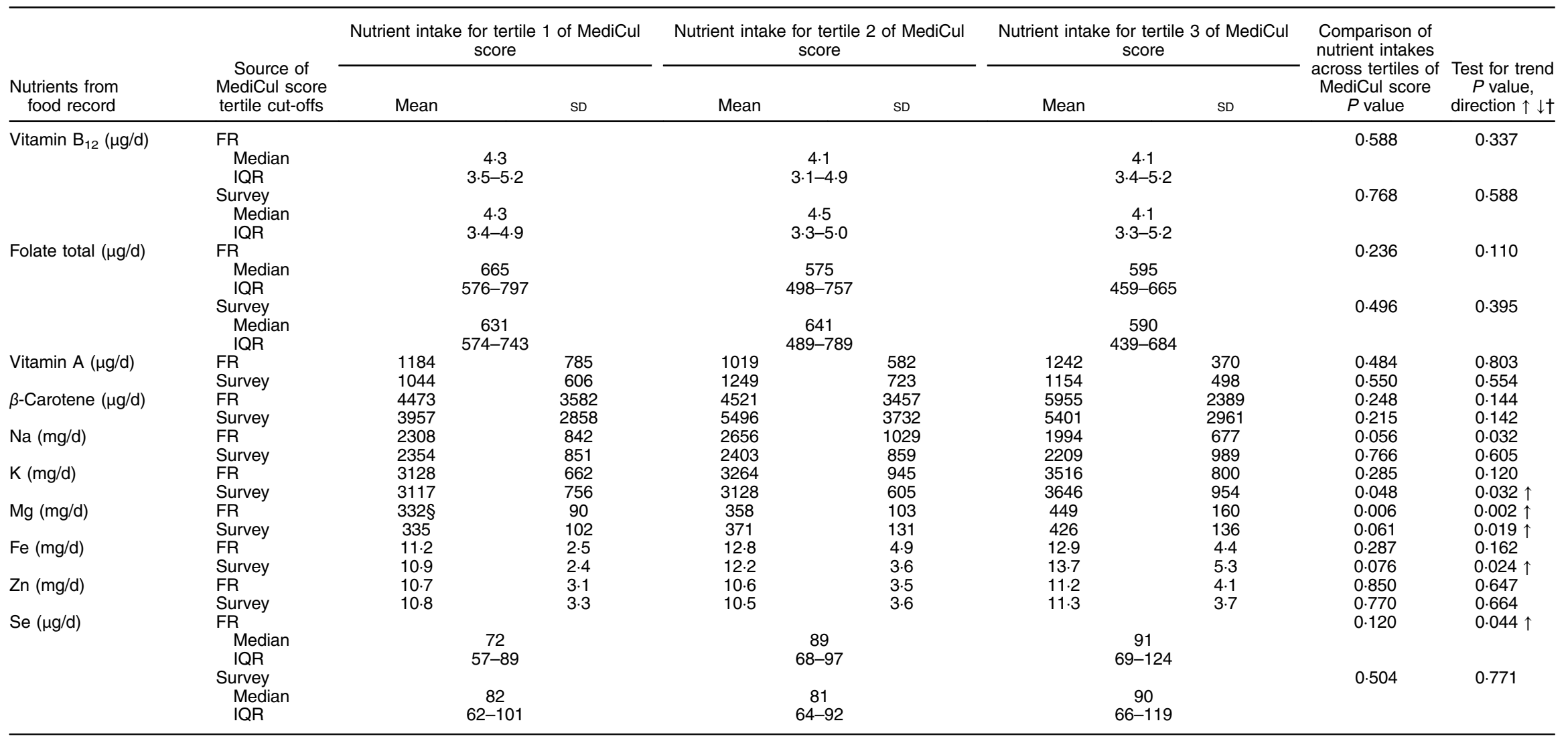

FR, food record; LC, long chain; ALA, $a$-linolenic acid; DPA, docosapentaenoic acid.

* Tertiles are derived for MediCul index scores from both the FR and survey A (first administration of MediCul). For survey A, the cut-offs for tertiles 2 and 3 were 47.0 and 58.0, respectively. Values are presented as means and standard deviations for normally distributed data or medians and IQR for non-normally distributed data. These data were normalised by logarithmic transformation for use in ANOVA models with the exception of alcohol and DHA, which were not for unequal variances. First (linear)- and second-(quadratic) order polynomial contrasts were applied to test for trends across tertiles, as well as line of best fit. In all cases, linear trends were significant, and there were no significan deviations from normality, except for Na when compared with tertiles from the FR and sugars when compared with tertiles from survey A, where the quadratic trend was positive and the most significant.

$\dagger$ Trend direction indicated as $\uparrow$ (increasing) or $\downarrow$ (decreasing).

‡ Significant differences between tertiles $1 \mathrm{v} .2$.

$\S$ Significant differences between tertiles $1 v$. 3 .

II Significant differences between tertiles 2 v. 3 . 
educated, may not be optimally protected by a Mediterranean dietary pattern, which is recommended for chronic disease prevention by $\mathrm{US}^{(51)}$ and Australian ${ }^{(52)}$ dietary guidelines and the National Health Service ${ }^{53)}$ in the UK. Future studies, however, are required to determine the direction of this relationship, as reverse causality is possible.

\section{Limitations}

Individual diets are complex and tend to vary over time, making measurement errors inevitable for all dietary methods ${ }^{(54)}$. The best methods for assessing populations at risk of dementia are yet to be elucidated ${ }^{(55)}$. The MediCul tool relies on self-reported data that could bias our results, especially given the cohort investigated. Yet there is limited research on cognitive status impact on the integrity of self-reported dietary data $^{(56)}$. One small study, including MCI participants of a similar age to our participants, found that cognitive impairment may inflate reliability and decrease validity of a $\mathrm{FFQ}^{(55)}$, which is not inconsistent with our findings. Further, MediCul has not yet been validated against disease risk factors and health outcomes or using biochemical measures of food intake, as has been reported for MEDAS ${ }^{(25,57-59)}$, and there has generally been limited use of biomarkers to investigate the relationship between diet and cognitive function ${ }^{(56)}$. However, this type of validation may be most relevant for the assessment of absolute nutrient intakes rather than an index for an overall dietary pattern. Although most FFQ solicit information about intake over the past year ${ }^{(22)}$, the MediCul tool asks participants about their last 6 months, which together with specific questions relating to cooking methods for both warmer and cooler weather may address some seasonal variation. However, this time period may still be problematic for information retrieval among individuals with MCI, although it has been reported that if the information recalled is considered inadequate, respondents rely on general knowledge of what they routinely eat $^{(56)}$. We also had a dietitian present, available to answer questions and check that responses were complete, limiting conclusions about other types of administrations or if the tool is entirely self-administered. Finally, the primary measure of global cognition (ADAS-Cog), assessed at the same time point for the whole cohort in our validity study, was taken, on average, 59 months earlier and it is possible that some participants may have reverted to normal cognition, inflating our results.

To reduce participant burden, we required only a 3-d FR using household measures, which is not ideal for foods that are not consumed daily. However, 3- to 4-d records appear acceptable as it has been reported that the validity of collected information decreases in the latter days of a 7 -d record, with recording periods of more than $4 \mathrm{~d}$ thought to be unsatisfactory owing to fatigue/disinterest, creating reactivity bias ${ }^{(22)}$. In common with most other indexes, no energy adjustment was made for age or sex, which is unavoidable with tools designed for easy use. The FoodWorks nutritional analysis programme has some limitations, with missing foods and categorisation used for some food groups; however, we adjusted for this manually. FoodWorks also contains Australian compositional data but this is unlikely to vary in ways that would influence reliability and validity of MediCul for use in other countries.

\section{Strengths}

Small-scale indexes such as screeners may not capture extreme levels of intakes, leading to over-estimation of associations with health outcomes ${ }^{(60)}$. More comprehensive surveys may also have higher validity ${ }^{(61)}$, although a ceiling of validity may exist $^{(54)}$. MediCul may be likened more to the larger-scale modified MedDietScore index tool, which has scoring from 0 to $130^{(62)}$, yet it is relatively quick to complete and compute scoring for, compared with a typical FFQ. MediCul also measures some unique aspects of Mediterranean cuisine such as high-moisture, lower-temperature cooking methods; frequent use of herbs and spices; and exposure to fermented foods such as olives. Such elements have been recommended to improve calculation of Mediterranean diet scores ${ }^{(63)}$. In its development, MediCul considered various best practice guidelines for dietary assessment ${ }^{(64)}$ now advised by The DIETary Assessment Tool NETwork ${ }^{(65)}$. The fact that a MEDAS score can also be derived from MediCul improves its utility so that comparisons with different studies, for various outcomes, can also be made.

\section{Conclusions}

Preventing or slowing cognitive decline may have a significant impact on the lives of individuals, families and carers, as well as future public health budgets. The Mediterranean diet is a promising lifestyle modality based on current evidence. Accurate measurement of adherence to the 'traditional' dietary pattern, among at-risk individuals or those with existing cognitive impairment, is vital to progress the field. We found that MediCul is a reliable and moderately valid tool to assess adherence to a Mediterranean dietary pattern among individuals with MCI who are at higher risk of converting to dementia. In our cohort of older Australians with MCI, the mean MediCul score was moderately low, suggesting poor compliance to this dietary pattern. MediCul may be a useful tool for future studies testing a Mediterranean diet intervention for various stages of cognitive decline, including MCI.

\section{Acknowledgements}

The authors thank Kenneth Daniel and Yian Noble for assistance with operationalising MediCul scoring. The authors are grateful to Fiona O'Leary and Lynne Cobiac for comments on a draft of the MediCul tool. The authors also acknowledge Adela Yip for help with data entry and greatly appreciate the involvement of all SMART participants.

The original SMART trial was funded by a National Health and Medical Research Council (NHMRC) of Australia Dementia Research Grant, project grant ID no. 512672 from 2008 to 2011. Additional funding for a research assistant position was provided by NHMRC Program grant ID no. 568969, and the project was supported by the University of Sydney and University of New South Wales. M. V. was supported by a University of New South Wales Vice Chancellor's Fellowship and consecutive 
NHMRC Clinical Career Development Fellowships. The original trial fulfilled a portion of the degree requirements for $\mathrm{PhD}$ for N. G. and C. S. This validity study did not receive financial support from any organisation and fulfilled a portion of the degree requirements for $\mathrm{PhD}$ for $\mathrm{S}$. R.-V.

The authors' contributions are as follows: S. R.-V. contributed to study design, data collection, data analyses, interpretation of findings and wrote the manuscript; S. R.-V. in conjunction with V. M. F. prepared the first draft of the MediCul tool; V. M. F. and M. A. F. S. contributed to study design, statistical analyses and interpretation of findings. M. A. F. S. and all other authors with the exception of S. R.-V. and V. M. F. contributed to the funding acquisition, study design and/or data collection/management and statistical analysis for the original SMART trial and the follow-up assessments of participants. All authors provided critical review and approved the final version of the manuscript.

The authors declare no personal or financial conflicts of interest.

\section{Supplementary materials}

For supplementary material/s referred to in this article, please visit https://doi.org/10.1017/S0007114518002428

\section{References}

1. Martinez-Gonzalez MA \& Bes-Rastrollo M (2014) Dietary patterns, Mediterranean diet, and cardiovascular disease. Curr Opin Lipidol 25, 20-26.

2. Estruch R, Ros E, Salas-Salvado J, et al. (2013) Primary prevention of cardiovascular disease with a Mediterranean diet. N Engl J Med 368, 1279-1290.

3. Tong TYN, Wareham NJ, Khaw K-T, et al. (2016) Prospective association of the Mediterranean diet with cardiovascular disease incidence and mortality and its population impact in a non-Mediterranean population: the EPIC-Norfolk study. $B M C$ Med 14, 135.

4. Gardener H, Wright CB, Gu Y, et al. (2011) Mediterraneanstyle diet and risk of ischemic stroke, myocardial infarction, and vascular death: the Northern Manhattan Study. Am J Clin Nutr 94, 1458-1464.

5. Couto E, Boffetta P, Lagiou P, et al. (2011) Mediterranean dietary pattern and cancer risk in the EPIC cohort. Br J Cancer 104, 1493-1499.

6. Toledo E, Salas-Salvadó J, Donat-Vargas C, et al. (2015) Mediterranean diet and invasive breast cancer risk among women at high cardiovascular risk in the PREDIMED trial: randomized clinical trial. JAMA Intern Med 175, 1752-1760.

7. Esposito K, Maiorino MI, Ceriello A, et al. (2010) Prevention and control of type 2 diabetes by Mediterranean diet: a systematic review. Diabetes Res Clin Pract 89, 97-102.

8. Salas-Salvadó J, Bulló M, Estruch R, et al. (2014) Prevention of diabetes with Mediterranean diets: a subgroup analysis of a randomized trial. Ann Intern Med 160, 1-10.

9. Psaltopoulou $\mathrm{T}$, Sergentanis $\mathrm{TN}$, Panagiotakos $\mathrm{DB}$, et al. (2013) Mediterranean diet, stroke, cognitive impairment, and depression: a meta-analysis. Ann Neurol 74, 580-591.

10. Sofi F, Macchi C, Abbate R, et al. (2013) Mediterranean diet and health status: an updated meta-analysis and a proposal for a literature-based adherence score. Public Health Nutr 17, 2769-2782.
11. Tangney CC, Kwasny MJ, Li H, et al. (2011) Adherence to a Mediterranean-type dietary pattern and cognitive decline in a community population. Am J Clin Nutr 93, 601-607.

12. Scarmeas N, Stern Y, Mayeux R, et al. (2006) Mediterranean diet, Alzheimer disease, and vascular mediation. Arch Neurol 63, 1709-1717.

13. Scarmeas N, Stern Y, Mayeux R, et al. (2009) Mediterranean diet and mild cognitive impairment. Arch Neurol 66, 216-225.

14. World Health Organization (2017) Rates of dementia. http:// www.who.int/en/news-room/fact-sheets/detail/dementia (accessed May 2018)

15. Bach A, Serra-Majem L, Carrasco JL, et al. (2006) The use of indexes evaluating the adherence to the Mediterranean diet in epidemiological studies: a review. Public Health Nutr 9, 132-146.

16. Milà-Villarroel R, Bach-Faig A, Puig J, et al. (2011) Comparison and evaluation of the reliability of indexes of adherence to the Mediterranean diet. Public Health Nutr 14, 2338-2345.

17. D'Alessandro A \& De Pergola G (2015) Mediterranean diet and cardiovascular disease: a critical evaluation of a priori dietary indexes. Nutrients 7, 7863-7888.

18. Ruiz AH, Garcia-Villanova B, Hernandez EJG, et al. (2015) Description of indexes based on the adherence to the Mediterranean dietary pattern: a review. Nutr Hosp 32, 1872-1884.

19. Radd-Vagenas S, Kouris-Blazos A, Fiatarone Singh M, et al. (2017) Evolution of Mediterranean diets and cuisine: concepts and definitions. Asia Pac J Clin Nutr 26, 749-763.

20. Schroder H, Fito M, Estruch R, et al. (2011) A short screener is valid for assessing Mediterranean diet adherence among older Spanish men and women. J Nutr 141, 1140-1145.

21. Cerwinske LA, Rasmussen HE, Lipson S, et al. (2017) Evaluation of a dietary screener: the Mediterranean Eating Pattern for Americans tool. J Hum Nutr Diet 30, 596-603.

22. Thompson FE \& Subar AF (2017) Chapter 1 - Dietary assessment methodology. In Nutrition in the Prevention and Treatment of Disease, 4th ed., pp. 5-48 [AM Coulston, CJ Boushey, MG Ferruzzi and LM Delahanty, editors]. London: Academic Press.

23. Trichopoulou A, Kouris-Blazos A, Wahlqvist ML, et al. (1995) Diet and overall survival in elderly people. BMJ 311, $1457-1460$

24. Trichopoulou A, Costacou T, Bamia C, et al. (2003) Adherence to a Mediterranean diet and survival in a Greek population. $N$ Engl J Med 348, 2599-2608.

25. Hebestreit K, Yahiaoui-Doktor M, Engel C, et al. (2017) Validation of the German version of the Mediterranean Diet Adherence Screener (MEDAS) questionnaire. BMC Cancer 17, 341 .

26. Sotos-Prieto M, Santos-Beneit G, Bodega P, et al. (2015) Validation of a questionnaire to measure overall Mediterranean lifestyle habits for research application: the MEDiterranean LIFEstyle index (MEDLIFE). Nutr Hosp 32, 1153-1163.

27. Freedman LS, Commins JM, Moler JE, et al. (2015) Pooled results from 5 validation studies of dietary self-report instruments using recovery biomarkers for potassium and sodium intake. Am J Epidemiol 181, 473-487.

28. Petersen RC (2004) Mild cognitive impairment as a diagnostic entity. J Intern Med 256, 183-194.

29. Petersen RC, Smith GE, Waring SC, et al. (1999) Mild cognitive impairment: clinical characterization and outcome. Arch Neurol 56, 303-308.

30. Valls-Pedret C, Sala-Vila A, Serra-Mir M, et al. (2015) Mediterranean diet and age-related cognitive decline: a randomized clinical trial. JAMA Intern Med 175, 1094-1103.

31. Martínez-González MA, García-Arellano A, Toledo E, et al. (2012) A 14-item Mediterranean diet assessment tool and 
obesity indexes among high-risk subjects: the PREDIMED trial. PLOS ONE 7, e43134.

32. Hernandez-Galiot A \& Goni I (2017) Adherence to the Mediterranean diet pattern, cognitive status and depressive symptoms in an elderly non-institutionalized population. Nutr Hosp 34, 338-344.

33. Gates NJ, Valenzuela M, Sachdev PS, et al. (2011) Study of mental activity and regular training (SMART) in at risk individuals: a randomised double blind, sham controlled, longitudinal trial. BMC Geriatr 11, 19.

34. Singh MAF, Gates N, Saigal N, et al. (2014) The Study of Mental and Resistance Training (SMART) study-resistance training and/or cognitive training in mild cognitive impairment: a randomized, double-blind, double-sham controlled trial. J Am Med Dir Assoc 15, 873-880.

35. Rosen WG, Mohs RC \& Davis KL (1984) A new rating scale for Alzheimer's disease. Am J Psychiatry 141, 1356-1364.

36. Hindmarch I, Lehfeld H, de Jongh $\mathrm{P}$, et al. (1998) The Bayer Activities of Daily Living Scale (B-ADL). Dement Geriatr Cogn Disord 9, Suppl. 2, 20-26.

37. Willett WC, Sacks F, Trichopoulou A, et al. (1995) Mediterranean diet pyramid: a cultural model for healthy eating. $A m J$ Clin Nutr 61, 1402S-1406S.

38. Oldways (2008) Mediterranean diet pyramid 2008. http:// oldwayspt.org/resources/heritage-pyramids/mediterraneanpyramid/overview (accessed May 2018).

39. Bach-Faig A, Berry EM, Lairon D, et al. (2011) Mediterranean diet pyramid today: science and cultural updates. Public Health Nutr 14, 2274-2284.

40. Australian Bureau of Statistics (2014) 4364.0.55.007 - Australian Health Survey: Nutrition First Results - Foods and Nutrients, 2011-12. Canberra: FSANZ.

41. Margetts BM \& Nelson MD (1997) Design Concepts in Nutritional Epidemiology, 2nd ed. New York: Oxford University Press.

42. Peat J (2001) Health Science Research: A Handbook of Quantitative Methods, 1st ed. Crows Nest: Allen \& Unwin.

43. Rosner B (2016) Fundamentals of Biostatistics, 8th ed. Boston, MA: Cengage Learning.

44. Bland JM \& Altman DG (1986) Statistical methods for assessing agreement between two methods of clinical measurement. Lancet i $\mathbf{i}, 307-310$.

45. Landis JR \& Koch GG (1977) The measurement of observer agreement for categorical data. Biometrics 33, 159-174.

46. Willett W (1998) Nutritional Epidemiology, 2nd ed., vol. 30. New York: Oxford University Press.

47. Klipstein-Grobusch K, Den Breeijen JH, Goldbohm RA, et al. (1998) Dietary assessment in the elderly: validation of a semiquantitative food frequency questionnaire. Eur $J$ Clin Nutr 52, 588-596.

48. Huybrechts I, Vereecken C, De Bacquer D, et al. (2010) Reproducibility and validity of a diet quality index for children assessed using a FFQ. Br J Nutr 104, 135-144.

49. Ambrosini GL, Van Roosbroeck SAH, Mackerras D, et al. (2003) The reliability of ten-year dietary recall: implications for cancer research. J Nutr 133, 2663-2668.
50. George ES, Kucianski T, Mayr HL, et al. (2018) A Mediterranean diet model in Australia: strategies for translating the traditional Mediterranean diet into a multicultural setting. Nutrients 10, 465.

51. US Department of Health and Human Services and US Department of Agriculture (2015) 2015-2020 Dietary Guidelines for Americans, 8th ed. Washington, DC: US Government Printing Office.

52. National Health and Medical Research Council (2013) Australian Dietary Guidelines. Canberra: National Health and Medical Research Council.

53. NHS (2017) What is a Mediterranean diet? https://www.nhs. uk/Livewell/Goodfood/Pages/what-is-a-Mediterranean-diet. aspx (accessed May 2018).

54. Willett W (2001) Invited commentary: a further look at dietary questionnaire validation. Am J Epidemiol 154, 1100-1102.

55. Bowman GL, Shannon J, Ho E, et al. (2011) Reliability and validity of food frequency questionnaire and nutrient biomarkers in elders with and without mild cognitive impairment. Alzheimer Dis Assoc Disord 25, 49-57.

56. Zuniga K \& McAuley E (2015) Considerations in selection of diet assessment methods for examining the effect of nutrition on cognition. J Nutr Health Aging 19, 333-340.

57. Díez-Espino J, Buil-Cosiales $\mathrm{P}$, Serrano-Martínez $\mathrm{M}$, et al. (2011) Adherence to the Mediterranean diet in patients with type 2 diabetes mellitus and HbA1c level. Ann Nutr Metab 58, $74-78$.

58. Sánchez-Taínta A, Estruch R, Bullo M, et al. (2008) Adherence to a Mediterranean-type diet and reduced prevalence of clustered cardiovascular risk factors in a cohort of 3204 highrisk patients. Eur J Cardiovasc Prev Rehabil 15, 589-593.

59. Schröder H, Marrugat J, Vila J, et al. (2004) Adherence to the traditional Mediterranean diet is inversely associated with body mass index and obesity in a Spanish population. $J$ Nutr 134, 3355-3361.

60. Panagiotakos DB, Pitsavos C \& Stefanadis C (2006) Dietary patterns: a Mediterranean diet score and its relation to clinical and biological markers of cardiovascular disease risk. Nutr Metab Cardiovasc Dis 16, 559-568.

61. Martinez-Gonzalez MA, Fernandez-Jarne E, Serrano-Martinez $\mathrm{M}$, et al. (2002) Mediterranean diet and reduction in the risk of a first acute myocardial infarction: an operational healthy dietary score. Eur J Nutr 41, 153-160.

62. Panagiotakos D, Kalogeropoulos N, Pitsavos C, et al. (2009) Validation of the MedDietScore via the determination of plasma fatty acids. Int J Food Sci Nutr 60, 168-180.

63. Hoffman R \& Gerber M (2013) Evaluating and adapting the Mediterranean diet for non-Mediterranean populations: a critical appraisal. Nutr Rev 71, 573-584.

64. Cade JE, Burley VJ, Warm DL, et al. (2004) Food-frequency questionnaires: a review of their design, validation and utilisation. Nutr Res Rev 17, 5-22.

65. Cade JE, Warthon-Medina M, Albar S, et al. (2017) DIET@NET: best practice guidelines for dietary assessment in health research. BMC Med 15, 202. 